\title{
Options for Using Test Data to Update Failure Stress
}

\author{
Erdem Acar ${ }^{1}$ \\ Mississippi State University, Mississippi State, MS, 39762 \\ Jungeun $\mathrm{An}^{2}$, Raphael T. Haftka ${ }^{3}$, Nam-Ho Kim ${ }^{4}$, and Peter J. Ifju ${ }^{5}$ \\ University of Florida, Gainesville, FL, 32611 \\ and \\ Theodore F. Johnson ${ }^{6}$ \\ NASA Langley Research Center, Hampton, VA, 23681
}

\begin{abstract}
In structural design, failure stresses are obtained from coupon tests and then used to predict failure under combined loads in structural elements. These tests are used to update the failure envelope of structural elements. In determining the failure stress of a single structural element, it is a common practice to replicate element tests and then use the lowest test result as a measure of conservativeness. This practice is equivalent to adding a knockdown factor to the stress allowable-one that is quite variable. Instead we propose using the average test result with an explicit knockdown factor and show that the variability is reduced and with it the likelihood of unconservative estimate of the failure stress. In addition, since failure theories are getting ever more accurate, it may not be reasonable to toss out analytical predictions and rely entirely on test results, which are subject to experimental error and variability. Instead, if the designer has some measure of confidence in the analytical predictions, Bayesian updating may further narrow down variability and decrease the chance of unconservative estimates of failure stresses. We demonstrate the gains associated with Bayesian updating for the case where the designer has only upper and lower bounds on the error in the analytical predictions. Examples with uniform and lognormal distributions of test results are used to compare the worst-test approach to the two alternatives with explicit knockdown factors. Both approaches yield large reductions in the likelihood of unconservative estimates of the failure stresses. The average approach reduced this likelihood by about a factor of two while the Bayesian approach by up to an order of magnitude (from $12.5 \%$ to $1 \%$ ). We also examine scenarios where estimates of error and variability are substantially off. We show that even then there are still substantial reductions in likelihood of unconservative estimates of failure stresses. Remarkably, the underestimate of variability also results in about $2 \%$ higher average of estimated failure stresses. Thus, we are able to simultaneously use higher average stress allowables (leading to lower weight) and reduce the likelihood of unconservative estimates!
\end{abstract}

Keywords: Knockdown factors, coupon tests, structural element tests, Bayesian statistics

\footnotetext{
${ }^{1}$ Postdoctoral Associate, the Center for Advanced Vehicular Systems, erdem@cavs.msstate.edu, Member, AIAA

${ }^{2} \mathrm{PhD}$ candidate, Mechanical and Aerospace Engineering Department, jean@ufl.edu, Student Member, AIAA

${ }^{3}$ Distinguished Professor, Mechanical and Aerospace Engineering Department, haftka@ufl.edu, Fellow, AIAA

${ }_{5}^{4}$ Professor, Mechanical and Aerospace Engineering Department, nkim@ufl.edu, Member, AIAA

${ }_{6}^{5}$ Professor, Mechanical and Aerospace Engineering Department, pgi@mae.ufl.edu, Member, AIAA

${ }^{6} \mathrm{PhD}$, Aerospace Engineer, NASA Langley Research Center, Theodore.F.Johnson@nasa.gov, Member, AIAA
} 


\section{Nomenclature}

$\left(\sigma_{f}\right) \quad=$ failure stress (a random variable)

$\left(\sigma_{f}\right)_{\text {calc }}=$ failure stress predicted by an engineer

$\left(\sigma_{f}\right)_{\text {true }}=$ the true failure stress of a structure

$\left(\sigma_{f}\right)_{\text {test }}=$ failure stress measured in tests

$\left(\sigma_{f}\right)_{\text {est }} \quad=$ estimated failure stress for three methods discussed in the paper

$\left(\sigma_{f}\right)_{i, \text { test }}=\mathrm{i}^{\text {th }}$ test result.

$\left(\sigma_{f}\right)_{\text {Bayes }}=$ failure stress calculated by Bayesian updating

$b_{e} \quad=$ error bounds

$e_{f} \quad=$ error of calculated failure stress w.r.t. the average true failure stress

$v_{f} \quad=$ variability in material properties

$\mathrm{k}_{\text {avg }}=$ knockdown factor used in average approach

$\mathrm{k}_{\text {Bayes }}=$ knockdown factor used in Bayesian approach

\section{Introduction}

EROSPACE structures have traditionally been designed using a deterministic approach based on the Federal Aviation Administration (FAA) or other regulations and guidelines. In deterministic approach, safety is achieved by combining safety factors with tests of material and structural components. On the other hand, there is growing interest in replacing safety factors by reliability-based design (e.g., Lincoln ${ }^{1}$, Wirsching ${ }^{2}$, SAE Aerospace Information Report $5080^{3}$ and Long and $\mathrm{Narciso}^{4}$ ). One step where probabilistic concepts have already taken place is in the choice of stress allowables. These are based on statistical analysis of coupon tests, with A-basis or B-basis allowables selected to be below $99 \%$ or $90 \%$ of the material populations, respectively, with $95 \%$ confidence.

After stress allowables are used to design structural elements, it is customary to test these elements in order to make sure that the failure predictions work well. This practice reduces the errors associated with applying engineering principles to measure the safety of structural elements.

Motivated by the remarkable safety level of current airliners, we analyzed the effects of measures that improve aircraft structural safety and compared the relative effectiveness of safety measures taken during aircraft structural design in our earlier works (Acar et al. ${ }^{5,6}$ ). The safety measures that we included in those works were the load safety factors of 1.5 , conservative material properties, redundancy, certification (or proof) test, and error and variability reduction. The most common form of error reduction is conducting structural element tests, which is the main focus of the present work. Structural element tests are usually used conservatively, by taking the worst result of a batch of nominally identical tests. This constitutes an implicit knockdown factor because of material and test-condition variability. However, the variability leads to excessive volatility in the knockdown factor and the resulting stress allowables. The main objective of the present paper is to show that simple statistical analysis may be used to replace the implicit knockdown factors with explicit ones and reduce the volatility in the stress allowables. This may be done with a minimum of statistical analysis by using the average test result instead of the worst one. Further improvements may be obtained by using the degree of confidence in the analytical estimates of the failure stress via Bayesian updating.

The present paper is organized as follows. Section 2 provides the analysis of the three options for using element test result to update the estimate of the failure envelope. Section 3 provides an illustrative example comparing the three approaches for one case of three element test results. Section 4 compares the three approaches for several examples, and Section 5 presents concluding remarks.

\section{Analysis}

Consider a typical situation relating to updating analytical predictions of strength based on a small number of nominally identical tests. We assume that the analytical prediction of the failure stress, $\left(\sigma_{f}\right)_{c a l c}$, applies to the average failure stress $\left(\bar{\sigma}_{f}\right)_{\text {true }}$ of an infinite number of nominally identical structures. The error $e_{f}$ of our analytical prediction is defined by Eq. (1).

$$
\left(\bar{\sigma}_{f}\right)_{\text {true }}=\left(1+e_{f}\right)\left(\sigma_{f}\right)_{\text {calc }}
$$

Here we assume that the designer can estimate the bounds $b_{e}$ (possibly conservative) on the magnitude of the error, and we further assume that the errors have a uniform distribution between the bounds. 


$$
f\left(e_{f}\right)=\left\{\begin{array}{ccc}
\frac{1}{2 b_{e}} & \text { if } & \left|e_{f}\right| \leq b_{e} \\
0 & & \text { otherwise }
\end{array}\right.
$$

That is, while the error has only one value in reality, the distribution reflects the uncertainty associated with our lack of knowledge of what this value is (so-called epistemic uncertainty). For simplicity we neglect experimental implementation errors and variability and measurement errors and variability and assume that the test results only reflect variability in material properties. In that case, the variability in material properties is defined by Eq. (3),

$$
\left(\sigma_{f}\right)_{\text {test }}=\left(1+v_{f}\right)\left(\bar{\sigma}_{f}\right)_{\text {true }}=\left(1+v_{f}\right)\left(1+e_{f}\right)\left(\sigma_{f}\right)_{\text {calc }}
$$

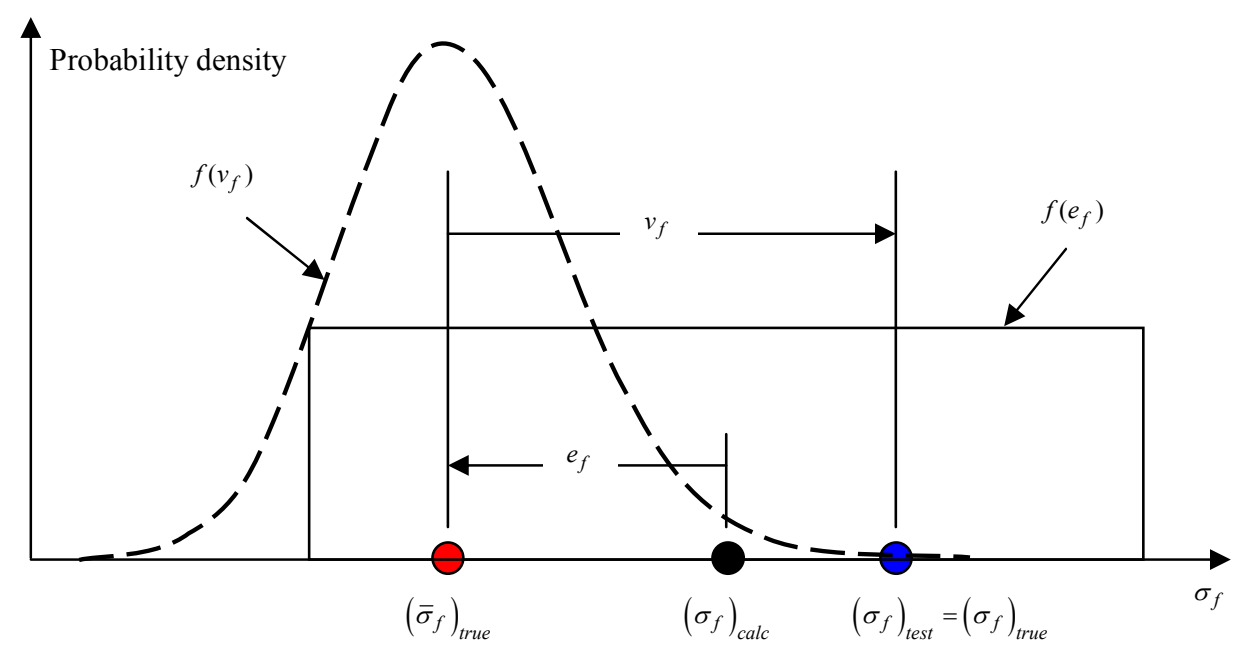

Figure 1: Error and variability in failure stress. The error is centered around the computed value, and is assumed to be uniformly distributed here. The variability distribution, on the other hand, is lognormal with mean equal to the true average failure stress.

Unlike the error, the variability does not reflect lack of knowledge but the randomness (so-called aleatory uncertainty) introduced into the failure stress by the manufacturing process for the material and the structure. This is depicted schematically in Fig. 1 for a uniform distribution of the error and a lognormal distribution of the variability.

The fact that experimental implementation errors and variability and measurement errors are not involved here is essentially equivalent to assuming that $\left(\sigma_{f}\right)_{\text {test }}=\left(\sigma_{f}\right)_{\text {true }}$. Here we use a lognormal distribution for the variability $v_{f}$, that is

$$
f\left(v_{f}\right)=L N\left(v_{f}+1 ; 0, \varsigma_{f}\right), \quad \varsigma_{f}=\sqrt{\ln \left(1+c_{f}^{2}\right)}
$$

where $c_{f}$ is the coefficient of variation (c.o.v). of the failure stress.

With an infinite number of tests, the average of test results would be $\left(\bar{\sigma}_{f}\right)_{\text {true }}$ and we could calculate $e_{f}$ exactly from Eq. (1). With a finite number of tests, we can obtain only an estimate of the error, and designers often opt for a conservative estimate.

We now consider three ways of estimating $\left(\bar{\sigma}_{f}\right)_{\text {true }}$.

1. Taking the lowest (worst) test result $\left(\bar{\sigma}_{f}\right)_{e s t}=\min \left(\sigma_{f}\right)_{i, \text { test }}$ where $\left(\sigma_{f}\right)_{i, \text { test }}$ is the result of the $i$ th test. This is often the approach taken by conservative designers. 
2. Taking the average test result and adding a knockdown factor $\mathrm{k}_{\text {avg }}$ in order to achieve the same average estimate of the failure stress obtained in the first approach. This may be viewed as achieving the same degree of conservatism associated with the first approach. That is,

$$
\left(\bar{\sigma}_{f}\right)_{\text {est }}=k_{\text {avg }}\left(\bar{\sigma}_{f}\right)_{\text {test }}
$$

3. Using the assumed initial distribution of the error in Eq. (2), we generate the corresponding probability distribution $f^{i n i}\left(\sigma_{f}\right)$ of the failure stress.

$$
f^{i n i}\left(\sigma_{f}\right)=\left\{\begin{array}{ccc}
\frac{1}{2 b_{e}\left(\sigma_{f}\right)_{\text {calc }}} & \text { if } & \left|\left(\sigma_{f}\right) /\left(\sigma_{f}\right)_{\text {calc }}-1\right| \leq b_{e} \\
0 & \text { otherwise }
\end{array}\right.
$$

Given $\left(\sigma_{f}\right)_{1, \text { test }}$, we use Bayesian updating to update the distribution as

$$
\begin{gathered}
f^{u p d}\left(\sigma_{f}\right)=\frac{f_{1, \text { test }}\left(\sigma_{f}\right) f^{i n i}\left(\sigma_{f}\right)}{\int_{-\infty}^{\infty} f_{1, \text { test }}\left(\sigma_{f}\right) f^{i n i}\left(\sigma_{f}\right) d \sigma_{f}} \\
\text { where } f_{1, \text { test }}\left(\sigma_{f}\right)=\operatorname{LN}\left(\left(\sigma_{f}\right)_{1, \text { test }} ; \sigma_{f}, \varsigma_{f}\right)
\end{gathered}
$$

Here $f_{1, \text { test }}\left(\sigma_{f}\right)$ is a function reflecting possible variability of the first test result $\left(\sigma_{f}\right)_{1, \text { test }}$. Note that it is not a probability distribution. Subsequent tests are handled by the same equations, using the updated distribution as the initial one. The advantage of the Bayesian updating approach is that it takes into account our confidence in the calculated failure stress. If our error bounds are very large, we can expect that this method would reduce to taking the average of the tests as we will see in Section 4. If the error bounds are very narrow, this approach will not allow the test results to change much the calculated stress, as these test results may reflect variability rather than error.

The average value of the last updated distribution is our Bayesian estimate of the average failure stress $\left(\bar{\sigma}_{f}\right)_{\text {Bayes }}$. Finally we add a knockdown factor $\mathrm{k}_{\text {Bayes }}$ in order to achieve the same degree of conservatism associated with the first approach. That is,

$$
\left(\bar{\sigma}_{f}\right)_{\text {est }}=k_{\text {Bayes }}\left(\bar{\sigma}_{f}\right)_{\text {Bayes }}
$$

\section{Illustrative Example}

To illustrate the use of Bayesian updating, we will consider the simpler case where the variability is also uniform rather than lognormal. So consider an example where the error bounds are $10 \%$, the variability is bounded by $15 \%$, and $\frac{\left(\sigma_{f}\right)_{i, \text { test }}}{\left(\sigma_{f}\right)_{\text {calc }}}=1.05,1.1,1.15 \quad i=1,2,3$. Furthermore, we will make the simplifying assumption that this $15 \%$ is of the calculated value, $\left(\sigma_{f}\right)_{\text {calc }}$, rather of the true value. Then, using Eq. (6) we can write

$$
f_{1, \text { test }}\left(\sigma_{f}\right)=\left\{\begin{array}{c}
\frac{1}{0.3\left(\sigma_{f}\right)_{\text {calc }}} \\
0
\end{array} \quad \text { if } \quad\left|\frac{\sigma_{f}-\left(\sigma_{f}\right)_{1, \text { test }}}{\left(\sigma_{f}\right)_{\text {calc }}}\right| \leq 0.15\right.
$$


and we will further assume that we chose our units so that $\left(\sigma_{f}\right)_{\text {calc }}=1$. In this case, the initial distribution of $f^{i n i}\left(\sigma_{f}\right)=5$ in $(0.9,1.1)$ and zero elsewhere. It is then very easy to check from Eq. (7) that the updated distribution is identical to the initial one because $f_{1, \text { test }}\left(\sigma_{f}\right)=1 / 0.3$ is constant in $(0.9,1.1)$. That is the first test does not provide any new information (Fig. 2.a). For the second test of 1.1, still $f_{1, \text { test }}\left(\sigma_{f}\right)=1 / 0.3$, but this time only in the interval $(0.95,1.1)$, so that the updated distribution is zero in $(0.9,0.95)$ (Fig. 2.b). Similarly the third test of 1.15 , excludes the interval $(0.95,1.0)$ (Fig. 2.c) so that finally we have a uniform distribution over $(1,1.1)$. The denominator of Eq. (7) will normalize the area to 1 , so that we get $f^{u p d}\left(\sigma_{f}\right)=10$ in $(1,1.1)$ and can use the average of 1.05 for our updated failure stress $\left(\bar{\sigma}_{f}\right)_{\text {Bayes }}$ (Fig. 2.d). Note that this is substantially different from taking the mean value of the tests, which is 1.1 . Indeed, if we expect that our calculation is correct to within $10 \%$, three tests whose average is 1.1 should not push us all the way towards this limit.
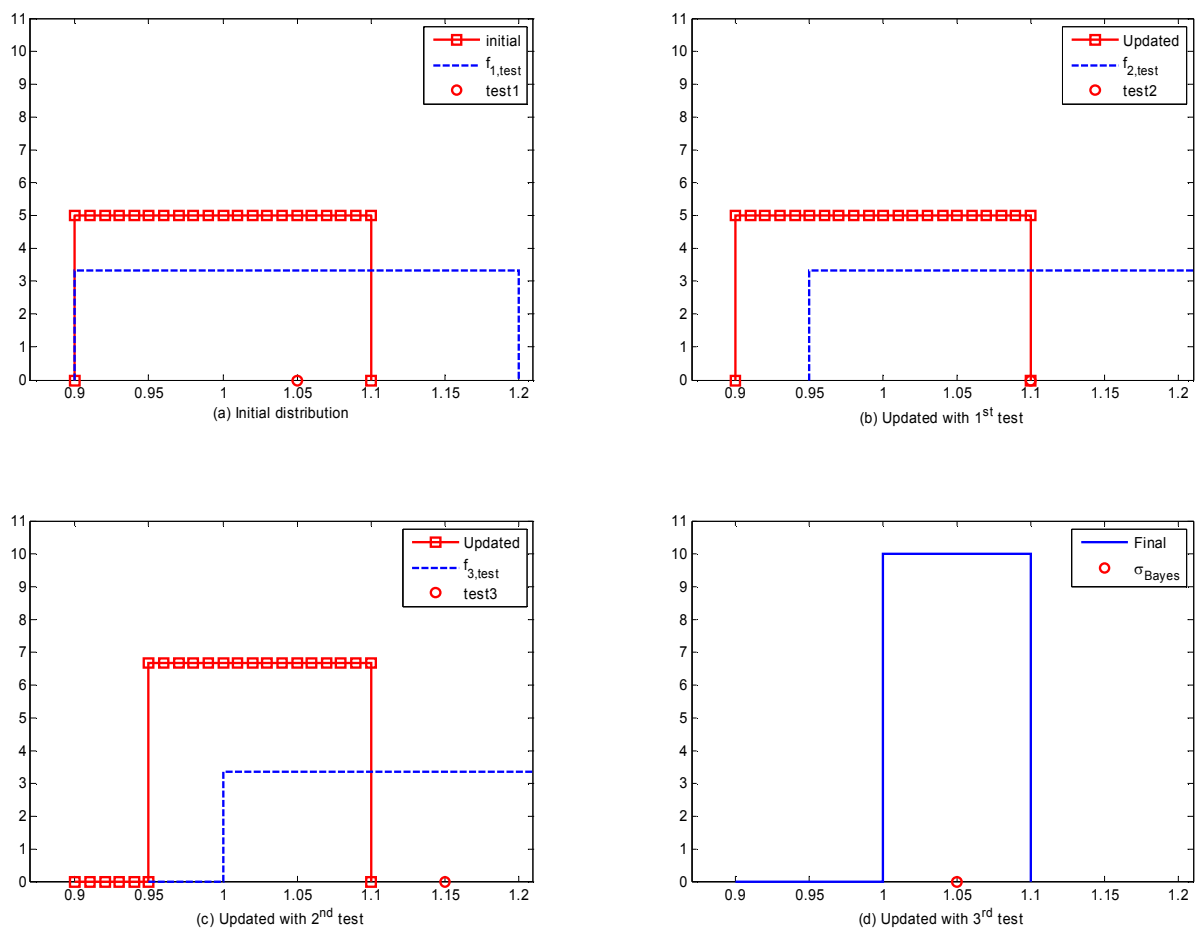

Figure 2: Illustratuve Example of Bayesian Updating. The initial distribution was uniform in [0.9,1.1] and the three tests were 1.05, 1.10, 1.15. The material variability is assumed to be governed by a uniform distribution with variability of $15 \%$ of the calculated value.

Next we have to ask, what knockdown factor we should have. We start by selecting a knockdown factor that will give the same average value of the estimated failure stress as the worst-load approach. The average is taken over all possible triads of test results, and the procedure for calculating the knockdown factors is discussed in Appendix A. For our uniform variability distribution, the value is 0.9250 . With the criterion of same average, the knockdown factors are the same for the average and Bayesian approaches, that is $k_{\text {Bayes }}=k_{\text {avg }}$ This means that if we use the average failure stress of 1.1 , the updated failure test should be $1.1 \times 0.925=1.0175$. With the Bayesian updating, the updated failure stress is $1.05 \times .925=0.9713$

Note that both results are lower than the worst of our three tests (1.05). This reflects the fact that when the average experimental result is higher than the calculated value, there is substantial chance that this is due to variability in material properties rather than to error. The average approach provides some compensation for this 
fact, while the Bayesian correction is more drastic because it takes into consideration our confidence in the analytical prediction of the failure stress.

For a more typical variability in failure stress we use a lognormal distribution with a coefficient of variation of $8 \%$. The evolution of distribution of the failure stress, $f\left(\sigma_{f}\right)$, is seen in Fig. 3. The average value of the final distribution is 1.0624, while the standard deviation of the final distribution is 0.02826. For the lognormal distribution, the knockdown factor is 0.9333 as shown in Appendix A. So to achieve the same degree of conservativeness with the average stress approach we will update the stress to 1.0266 , and with the Bayesian approach to 0.9915 .

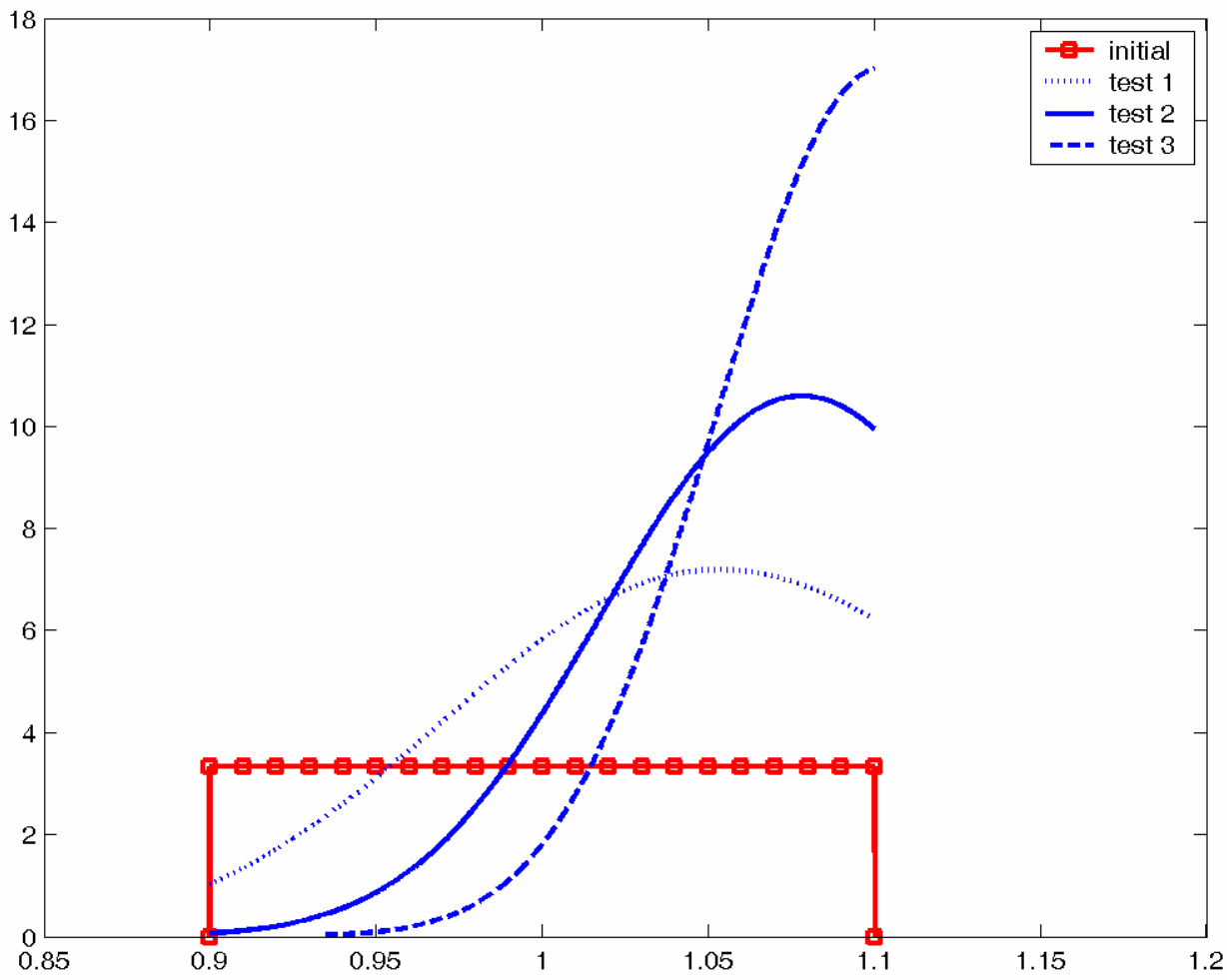

Figure 3. Evolution of failure stress distribution, $f\left(\sigma_{f}\right)$, with Bayesian updating. The initial distribution was uniform in $[0.9,1.1]$ and the three tests were $1.05,1.10,1.15$. The material variability is assumed to be governed by a lognormal distribution with a coefficient of variation of $8 \%$.

\section{Variability in test results and its effect on conservativeness}

The most important advantage of the average and Bayesian approaches over the worst-value approach is that they temper the variability in test results. These tests are intended to help us reduce the error in analytical predictions of failure stresses. However, the variability in material properties contaminates the test results and reduces their value for correcting the predictions. As a result, there is a chance that the corrected failure stress is unconservative.

For the example with the $15 \%$ uniformly distributed variability, the standard deviation of the worst test result was estimated by Monte Carlo simulation to be 0.058 or $6.3 \%$ of the mean of the worst result. As shown in Appendix B, there is $12.5 \%$ chance that all three tests will give unconservative estimate of the failure stress, so that the worst one will also be unconservative. On the other hand, the standard deviation of the average test result is 0.05 , which is $5 \%$ of the mean of the average test result. That is, by using the average test result with a knockdown factor of 0.925 , we achieve the same degree of conservativeness on average but with $20 \%$ less volatility. The analytical prediction in Appendix B shows that this reduces the chance of unconservative estimate to $5.4 \%$, less than half that 
of the worst-test approach. With the Bayesian approach, Monte Carlo simulations reduce the standard deviation of the estimated failure strain to $3.2 \%$, and drastically reduce the chance of an unconservative estimate to about $1 \%$.

Not only is the percentage of unconservative estimates reduced, but the average error in the unconservative estimates is also reduced. It is also shown in Appendix B that for the worst-case approach the average over estimate of failure stresses for the $12.5 \%$ unconservative results is $3.75 \%$. For the $5.4 \%$ unconservative results using the mean test result, the average over estimate is only $1.6 \%$, and for the $1 \%$ unconservative results in the Bayesian approach the average over estimate is only $0.6 \%$.

Table 1 summarizes the above results and provides the corresponding results for the lognormal distribution. The table also shows results (in parentheses) based on $15 \%$ confidence interval in the analytical predictions. We see that the results are similar for the lognormal distribution. For confidence intervals of $+-10 \%$ the likelihood of unconservative estimate of the failure stress is reduced dramatically. For $+-15 \%$ confidence interval, the results are as expected closer to the average, but still provide significant reduction in the chance for over estimating the failure stress.

Table 1. Comparison of coefficient of variation (COV) and likelihood of unconservative estimates using worst-case, average, and Bayesian approaches. Nominal variability case. (The Bayesian approach is based on a $10 \%$ confidence in the calculated result with uniform distribution in $[0.9,1.1])$. The numbers in parenthesis are for $15 \%$ confidence intervals.

\begin{tabular}{c|ccc|ccc}
\hline \multirow{2}{*}{ Variability } & \multicolumn{3}{|c|}{ Uniform (+-15\% bounds) } & \multicolumn{3}{c}{ Lognormal (8\% Cov) } \\
\cline { 2 - 7 } & Worst & Average & Bayesian & Worst & Average & Bayesian \\
\hline \% COV & 6.3 & 5.0 & $3.2(3.6)$ & 5.9 & 4.6 & $3.3(3.7)$ \\
\hline Mean & 0.925 & 0.925 & 0.925 & 0.9333 & 0.9333 & 0.9333 \\
\hline \% Unconservative & 12.5 & 5.4 & $1.0(2.5)$ & 11.3 & 6.4 & $2.8(4.3)$ \\
\hline Average & 3.75 & 1.6 & $0.6(1.3)$ & 2.7 & 2.1 & $1.3(1.8)$ \\
unconservative, \% & & & & & & \\
\hline
\end{tabular}

\section{Effect of uncertainty about uncertainty}

The calculations in Section 4 represent an ideal case, where we assumed that we have good error bounds and we know exactly the variability distribution of test results to apply with Bayesian updating and to set the explicit knockdown factor. In practice we may tend to select conservative error bounds, and conservative estimates of variability to allow for variability introduced by the experimental procedure. In this section we will therefore consider the following scenarios:

a. Error bounds are increased from $10 \%$ to $15 \%$.

b. The variance bounds are increased from $15 \%$ to $20 \%$ for the uniform distribution and from $8 \%$ to $10 \%$ for the lognormal distribution. Note that latter increase would correspond to an additional $6 \%$ variability from an independent source like testing variability on top of the $8 \%$ material variability. However, the explicit knockdown factors are left at their previous values of 0.925 , and 0.933 , respectively.

c. Combination of both error bound and variance increases.

Table 2: Comparison of coefficient of variation (COV) and likelihood of unconservative estimates using worst-case, average, and Bayesian approaches. Increased variability case. (The Bayesian approach is based on a $10 \%$ confidence in the calculated result with uniform distribution in $[0.9,1.1])$. The numbers in parenthesis are for $15 \%$ confidence intervals.

\begin{tabular}{c|ccc|ccc}
\hline \multirow{2}{*}{ Variability } & \multicolumn{3}{|c|}{ Uniform (+-20\% bounds) } & \multicolumn{3}{c}{ Lognormal (10\% Cov) } \\
\cline { 2 - 7 } & Worst & Average & Bayesian & Worst & Average & Bayesian \\
\hline \% COV & 8.6 & 6.66 & $3.6(4.4)$ & 7.4 & 5.8 & $3.8(4.4)$ \\
\hline Mean & 0.9 & 0.925 & 0.925 & 0.9172 & 0.9333 & 0.9333 \\
\hline \% Unconservative & 12.5 & 11.8 & $2.2(5.3)$ & 11.1 & 6.4 & $4.6(7.3)$ \\
\hline Average & 5.0 & 2.75 & $0.7(1.6)$ & 3.3 & 2.7 & $1.7(2.1)$ \\
unconservative, \% & & & & & & \\
\hline
\end{tabular}

Scenario (a) was discussed in the previous section, and was shown in Table 1 (in parenthesis). The table showed that being conservative and overestimating the error by $50 \%$ reduces the advantage of the Bayesian approach, but 
still leaves a substantial margin of improvement. Compared to the worst-test approach the probability of unconservative estimate of the failure stress is still reduced by a factor of 2.6 to 5 .

The results corresponding to (b) and (c) are summarized in Table 2. It is important to note that being conservative on the variability could entail using a lower knockdown factor which would make the Bayesian approach overly conservative if that variance was overestimated. Accordingly, we show results using the knockdown factors corresponding to the lower variability. Consequently, the mean value of the Bayesian estimated stresses is higher for these scenarios. For the uniform distribution the mean is 0.925 compared to 0.9 , which means that with the Bayesian approach we will also be able to use stress allowable that are higher by about $2.7 \%$. This is likely to correspond to similar reduction in weight. For the lognormal distribution the corresponding values are 0.9333 and 0.9172 , or $1.7 \%$ gain.

For scenario (b) where only the variability is underestimated, we achieve the higher stress allowable with a reduction of more than a factor of five in the likelihood of having unconservative failure stress estimates $(2.2 \%$ compared to $12.5 \%)$ for the uniform distribution and better than a factor of two (4.6\% compared to $11.1 \%)$ for the lognormal distribution. Furthermore, these gains in stress allowables and reductions in likelihood of unconservative estimates of failure stresses are accompanied by substantial reductions in the magnitude of the average unconservative error: from $5 \%$ to $0.7 \%$ for the uniform distribution and from $3.3 \%$ to $1.7 \%$ for the lognormal distribution.

For scenario (c), which combines overestimate of the error with underestimate of the variability gains are diminished but are still substantial. For the uniform distribution, gains in stress allowables are still accompanied by better than a factor of two reduction in the likelihood of unconservative estimates (5.3\% compared to $12.5 \%)$ for the uniform distribution and almost a factor of two for the lognormal distribution $(7.3 \%$ compared to $11.1 \%)$.

\section{Concluding remark}

Test results of structural elements are often used in a conservative fashion by updating estimates of failure stress based on the results of the worst test. This constitutes an implicit knockdown factor. In this paper we show that the variability implicit in this approach makes it inefficient compared to using less variable estimates of the failure stress and adding an explicit knockdown factor. We compared two alternative approaches to the worst-case approach: one based on average test results and one based on Bayesian update of an assumed error distribution.

Examples with uniform and lognormal distributions of test results were used to compare the worst-test approach to the two alternatives with explicit knockdown factors. Both approaches were shown to yield large reductions in the likelihood of unconservative estimates of the failure stresses. The average approach reduced this likelihood by about a factor of two while the Bayesian approach by up to an order of magnitude (from $12.5 \%$ to $1 \%$ ). In addition, the magnitude of the average lack of conservativeness was also greatly reduced.

We next examined scenarios where the estimates of error and variability are substantially in error. Specifically we considered a scenario where error estimates are inflated from $10 \%$ to $15 \%$. Then we considered a scenario where experimental errors are almost as high as material variability, increasing the combined coefficient of variation from $8 \%$ to $10 \%$. Finally we considered a scenario combining both inflated error estimates and underestimated experimental variability. We showed that even under these adverse conditions there are still substantial gains in reduced likelihood of unconservative estimates of failure stresses. Remarkably, the underestimate of variability also results in about $2 \%$ higher average of estimated failure stresses. Thus, we are able to simultaneously use higher average stress allowables and reduce the likelihood of unconservative estimates!

\section{Appendix A: Explicit Knockdown Factors}

In the application process of average and Bayesian approach explained in this paper, an explicit knockdown factor is needed that corresponds to the same average estimated failure stress as the worst approach on structural tests. With three nominally identical tests, the worst result will follow an extreme value distribution. So, the knockdown factors used in this paper $\left(\mathrm{k}_{\mathrm{avg}}, \mathrm{k}_{\text {Bayes }}\right)$ are calculated using extreme value distribution. The procedures are as follows:

1. If $f(x)$ is a uniform PDF in $[0.85,1.15]$, then the corresponding CDF $F(x)$ is a linear function in Eq. (10).

$$
F_{X}(x)=\frac{1}{0.3}(x-0.85)
$$

2. The cumulative distribution of the worst of three tests can be expressed by Eq. (11), 


$$
F_{X_{1}^{3}}(x)=1-\left(1-F_{X}(x)\right)^{3}
$$

where subscript 1 stands for a minimum and superscript 3 on LHS means our sample size.

3. We can calculate the mean value of worst three results from Eq. (12).

$$
\begin{aligned}
\text { mean } & =\int_{-\infty}^{\infty} x f_{X_{1}^{3}}(x) d x=\int_{0.85}^{1.15} x f_{X_{1}^{3}}(x) d x \\
& =\left.x F_{X_{1}^{3}}(x)\right|_{0.85} ^{1.15}-\int_{0.85}^{1.15} F_{X_{1}^{3}}(x) \\
& =1.15-0.225=0.9250
\end{aligned}
$$

4. If $f(x)$ is a lognormal pdf, then $F_{X}(x)$ can be defined by Eq. (13), a standard form of lognormal distribution.

$$
F_{X}(x)=\frac{1}{2}\left[1+\operatorname{erf}\left(\frac{\ln x}{\varsigma_{f} \sqrt{2}}\right)\right]
$$

Using the worst case distribution in Eq. (11) and mean calculation in Eq. (12), we can calculate the mean of worst of three results to be 0.9333 .

\section{Appendix B: Analytical Prediction of Variability on Worst and Average Cases}

\section{A. Worst case approach}

If we have three test results having same types of probability distribution, the worst of the test result will follow an extreme distribution given in Eq. (11). Exact numerical evaluations for the worst-case approach in table 1 are presented.

\section{Uniformly distributed samples}

For test results with $+-15 \%$ variability uniformly distributed, the CDF is given in Eq. (10). The measure of unconservative ratio, average unconservative ratio, and coefficient of variation (c.o.v.) are calculated by Eq. (14).

$$
\begin{aligned}
\text { unconservative } & =\int_{x_{\text {true }}}^{\infty} f_{X_{1}^{3}}(x) d x \\
\text { Average unconservative } & =\int_{x_{\text {true }}}^{\infty} x f_{X_{1}^{3}}(x) d x-x_{\text {true }} \\
\text { c.o.v. } & =\frac{\sqrt{\int_{-\infty}^{\infty} x^{2} f_{X_{1}^{3}}(x) d x-\text { mean }^{2}}}{\text { mean }}
\end{aligned}
$$

The PDF $f_{X_{1}^{3}}(x)$ can be obtained by differentiating Eq. (11) with respect to $x$.

The unconservativeness of applying worst case approach is $12.5 \%$, average unconservativeness is $3.75 \%$, and the c.o.v. is $6.28 \%$.

2. Lognormally distributed samples

Using Eq. (10) and applying $F_{X}$ defined as the CDF of a lognormal distribution function given by Eq (13), we can derive $F_{X_{1}^{3}}(x)$ and $f_{X_{1}^{3}}(x)$. By Eq. (14), we can also calculate the unconservativeness is $11.34 \%$, average unconservativeness is $2.72 \%$, and the c.o.v. of the distribution is $5.92 \%$.

\section{B. Average approach}

The probability distribution of mean of the three test results can be calculated by using sum distribution on three identical probability distribution functions. 


\section{Uniformly distributed samples}

Uniform sum distribution ${ }^{7}$ is a well known distribution function to represent a sum of more than two uniform random variables. Figure 4 and Eq. (15) shows the analytical distribution of the mean value of three tests having uniform variability.

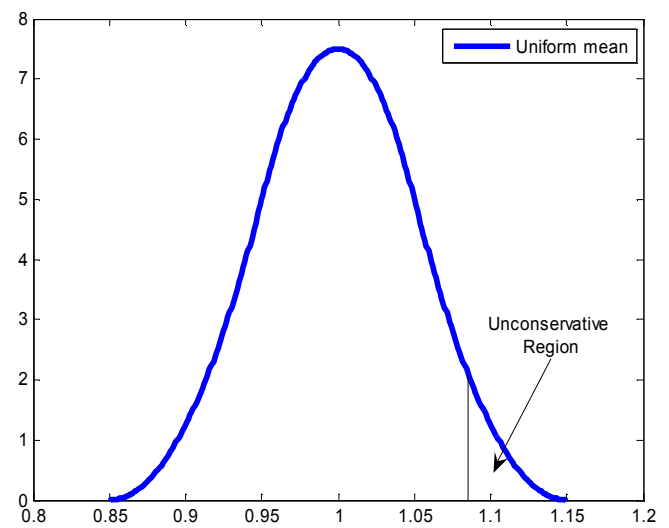

Figure 4. Uniform sum distribution $(n=3)$

$$
\begin{aligned}
f(x)= & \frac{1}{4 d^{3}}\left[-\left(-b_{4}+x\right)^{2} \operatorname{sgn}\left(-b_{4}+x\right)+3\left(-b_{3}+x\right)^{2} \operatorname{sgn}\left(-b_{3}+x\right)\right. \\
& \left.-3\left(-b_{2}+x\right)^{2} \operatorname{sgn}\left(-b_{2}+x\right)+\left(-b_{1}+x\right)^{2} \operatorname{sgn}\left(-b_{1}+x\right)\right]
\end{aligned}
$$

where $b_{i}(\mathrm{i}=1,2,3,4)$ are $0.85,0.95,1.05,1.15$, respectively and $d=0.1$, and $\operatorname{sgn}()$ takes the values of $-1,1$ or 0 when the value inside the parentheses is negative, positive or zero, respectively.

The final failure stress estimate is determined after adding $\mathrm{k}_{\text {avg }}$ to this distribution. We have unconservative region as in figure 4. Applying Eq. (15) in the place of $f_{X_{1}^{3}}(x)$ in Eq. (14), we can obtain $5.45 \%$ unconservativeness, $1.59 \%$ average unconservativeness, and the c.o.v., which is exactly $5.0 \%$.

\section{Acknowledgments}

The authors gratefully acknowledge the support of the research by the NASA center and the United States Air Force, Air Force Research Laboratory, under award FA9550-07-1-0018 (Dr. Victor Giurgiutiu, Program Manager).

\section{References}

${ }^{1}$ Lincoln, J.W., "Method for Computation of Structural Failure Probability for an Aircraft," ASD-TR-80-5035, WrightPatterson Air Force Base, OH, July 1980.

${ }^{2}$ Wirsching, P.H., "Literature Review on Mechanical Reliability and Probabilistic Design," Probabilistic Structural Analysis Methods for Select Space Propulsion System Components (PSAM), NASA Contractor Report 189159, Vol. III, Washington, D.C., 1992.

${ }^{3}$ Society of Automotive Engineers (SAE), "Integration of Probabilistic Methods into the Design Process,", Aerospace Information Report 5080, Warrendale, PA, 1997.

${ }^{4}$ Long, M.W., and Narciso. J.D., "Probabilistic Design Methodology for Composite Aircraft Structures," FAA Final Report, DOD/FAA/AR-99/2, Washington, D.C., June 1999.

${ }^{5}$ Acar, E., Kale, A., Haftka, R.T., and Stroud, W.J., "Structural Safety Measures for Airplanes," Journal of Aircraft, Vol. 43, No. 1, 2006, pp. 30-38.

${ }^{6}$ Acar, E., Kale, A., and Haftka, R.T., "Comparing Effectiveness of Measures that Improve Aircraft Structural Safety," to appear, ASCE Journal of Aerospace Engineering, Vol. 20, No.3, July 2007.

${ }^{7}$ Weisstein, Eric W. "Uniform Sum Distribution," MathWorld--A Wolfram Web Resource. URL: http://mathworld.wolfram.com/UniformSumDistribution.html [cited 2 March 2007]. 